\title{
Foreword
}

\section{Device-Based Arrhythmia Monitoring}

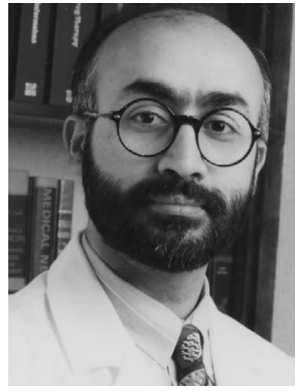

Ranjan K. Thakur, MD, MPH, MBA, FHRS

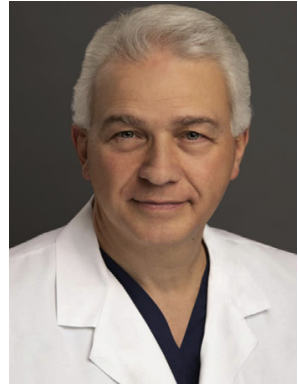

Andrea Natale, MD, FACC, FHRS

Editors

We are pleased to welcome Drs Mittal and Slotwiner as coeditors of this issue of the Cardiac Electrophysiology Clinics devoted to the advances in Device-Based Arrhythmia Monitoring.

Arrhythmia monitoring started with the advent of the ambulatory 24-hour Holter monitoring and then advanced to longer-term (30 days) monitoring. Advances in technology enabled cardiac implantable electronic device (pacemaker and defibrillator)-based arrhythmia surveillance and the implantable loop recorder, originally envisioned for diagnosis of unexplained syncope, but indications quickly expanded to include suspected arrhythmias and cryptogenic stroke to look for paroxysmal atrial fibrillation.

While technological advances have been tremendous, by enabling these developments, we are at an inflection point in technological innovation fueled by development of 5-G and artificial intelligence. The future is bright and limited only by imagination. Future developments will expand technologyassisted diagnostic prowess, expand remote device programming, and increase patient convenience, as the new generation of tech-savvy patients will demand it and change workflows in our clinics. Wearable cardiac and arrhythmia monitors will have an impact on diagnosis and monitoring of arrhythmias. Many of these technological developments are too recent to ascertain what role it may have and how they may be utilized. Many of these advances will have to be balanced with the challenges posed by cybersecurity and legal issues.

Drs Mittal and Slotwiner have assembled a group of experts from academia and industry who have a focus on these issues of arrhythmia monitoring, and the articles in this issue of the Cardiac Electrophysiology Clinics will update the readers on the developments in this field, contemporary issues, and what the future may hold. We congratulate them for a unique summary of these issues in arrhythmia monitoring.

Ranjan K. Thakur, MD, MPH, MBA, FHRS Sparrow Thoracic and Cardiovascular Institute Michigan State University 1440 East Michigan Avenue; Suite 400 Lansing, MI 48912, USA

Andrea Natale, MD, FACC, FHRS Texas Cardiac Arrhythmia Institute

Center for Atrial Fibrillation at

St. David's Medical Center 1015 East 32nd Street, Suite 516

Austin, TX 78705, USA

E-mail addresses:

thakur@msu.edu (R.K. Thakur) andrea.natale@stdavids.com (A. Natale) 